\title{
Safety assessment of sorafenib in Chinese patients with unresectable hepatocellular carcinoma: subgroup analysis of the GIDEON study
}

Sheng-Long Ye ${ }^{1 *}$, Jiamei Yang ${ }^{2}$, Ping Bie ${ }^{3}$, Shuijun Zhang ${ }^{4}$, Xiaoping Chen ${ }^{5}$, Fengyong Liu ${ }^{6}$, Luming Liu', Jie Zhou ${ }^{8}$, Kefeng Dou ${ }^{9}$, Chunyi Hao ${ }^{10}$, Guoliang Shao ${ }^{11}$, Qiang Xia ${ }^{12}$, Yajin Chen ${ }^{13}$, Jijin Yang ${ }^{14}$, Xiaxing Deng ${ }^{15}$, Yunpeng Liu ${ }^{16}$, Yunfei Yuan ${ }^{17}$, Zhiren Fu ${ }^{18}$, Keiko Nakajima ${ }^{19}$ and Zhengguang Lv ${ }^{20}$

\begin{abstract}
Background: This study aimed to investigate the safety of sorafenib for the treatment of unresectable hepatocellular carcinoma in Chinese patients.

Methods: A subgroup of 345 Chinese patients from the international database of the Global Investigation of therapeutic DEcisions in hepatocellular carcinoma and Of its treatment with sorafeNib (GIDEON) study was included in this analysis. Safety assessment measures were adverse events (AEs) and serious adverse events (SAEs) graded using the National Cancer Institute Common Terminology Criteria version 3.0.

Results: Of 331 evaluable patients, 98\% started sorafenib at $800 \mathrm{mg} /$ day. The median treatment duration was 22 weeks (range, 0.1-116 weeks), and median overall survival (OS) was 322 days (10.7 months). Approximately $50 \%$ of patients had at least one adverse event, and $6 \%$ had grade 3-4 adverse events. Drug-related adverse events were experienced by $29 \%$ of patients, and 3.6\% had grade 3-4 drug-related adverse events. Overall, $23 \%$ of patients $(n=77)$ experienced serious adverse events, among which only 1 event was drug-related (0.3\%). No differences in overall adverse events, serious adverse events, and deaths were observed between Child-Pugh A and Child-Pugh B patients. The most frequent drug-related adverse events were dermatological/ skin (24\%), hand-foot skin reaction (20\%), gastrointestinal (11\%), and diarrhea (11\%). The majority of adverse events occurred within 30 days of beginning sorafenib.
\end{abstract}

Conclusion: Sorafenib has satisfactory efficacy and safety in Chinese Child-Pugh A and B patients with unresectable HCC using the recommended dosage of $800 \mathrm{mg} /$ day, and the safety of sorafenib is not affected by liver function. Prophylaxis for gastrointestinal adverse events may help to decrease dose interruptions or discontinuation.

Trial registration: ClinicalTrials.gov; Identifier: NCT00812175. Date of registration: December 19, 2008.

\footnotetext{
* Correspondence: slye@shmu.edu.cn

'Liver Cancer Institute, Zhongshan Hospital, Fudan University, 136 Yixueyuan

Rd, Shanghai 200032, China

Full list of author information is available at the end of the article
} 


\section{Background}

Hepatocellular carcinoma (HCC) is the second leading cause of cancer-related deaths worldwide, and the incidence is increasing with 782,000 cases diagnosed worldwide in 2012 [1]. The incidence of HCC is highest in the East and South-East Asia, with China accounting for nearly half of all HCC cases and deaths globally [2]. In Asian countries, the main HCC treatment options include surgical resection, ablation, transarterial chemoembolization (TACE), and radiation or chemotherapy based on liver function $[3,4]$. Surgical resection may offer a 5-year survival up to $70 \%$ in patients with excellent liver function but for patients with advanced disease, surgical resection is an option for less than $20 \%$ of patients [5]. While TACE may provide a meaningful increase in overall survival (OS) for some patients with intermediate (unresectable) disease, many patients in the Asia-Pacific region present with advanced HCC for which most therapies do not provide a meaningful increase in OS [6].

Sorafenib (Nexavar ${ }^{\oplus}$, a registered trademark of Bayer HealthCare Pharmaceuticals, Ltd., China) is an oral multikinase inhibitor of vascular endothelial growth factor receptor and platelet-derived growth factor receptor that inhibits tumor-cell proliferation and tumor angiogenesis $[7,8]$. A number of studies examining the safety and efficacy of sorafenib in HCC, both alone, and in combination with conventional TACE, have been conducted globally [9-13], and specifically in the Asia-Pacific region [14-17]. The Sorafenib Hepatocellular Carcinoma Assessment Randomised Protocol (SHARP) trial, an international, multi-centered, phase III placebo-controlled study of 602 patients with advanced HCC, found that sorafenib significantly prolonged survival in patients with unresectable HCC, with an overall survival of 10.7 months compared to 7.9 months for placebo, with an acceptable safety profile [13]. The 2009 Asia-Pacific (AP) study showed that sorafenib was well tolerated and demonstrated a significant improvement in OS over placebo in the treatment of HCC in Asian patients [14].

The Global Investigation of therapeutic DEcisions in hepatocellular carcinoma and Of its treatment with sorafeNib (GIDEON) trial, a non-interventional surveillance study, evaluated the safety and efficacy of sorafenib in patients with unresectable HCC in real-world practice [18]. The GIDEON trial generated a large database of HCC patients (approximately 3000) across different disease subclasses and stages, in different regions. Two interim analyses $[19,20]$ reported global and regional differences in patient baseline characteristics, disease etiology, real-world practice patterns, and treatment outcomes. Data from China in the GIDEON database provides the ability to examine the effects of sorafenib in a Chinese population. Liu et al. [21] examined sorafenib and TACE in the GIDEON Chinese subgroup and found that sorafenib was usually administered in patients with tumor progression or poor liver function after TACE and that survival outcomes were still considered satisfactory. Ye et al. [22] also studied the GIDEON Chinese subgroup and reported that earlier administration of sorafenib may improve outcomes in patients with unresectable HCC and portal vein tumor thrombosis.

Sorafenib is typically administered at a dose of $400 \mathrm{mg}$ twice a day; however, in practice dosing varies based on physician preference, Child-Pugh class, performance status, and comorbidities [23, 24]. Few studies have reported sorafenib efficacy and safety with respect to dosing and Child-Pugh class in HCC patients. Analysis of the GIDEON data indicated that the median overall survival (OS) was longer for Child-Pugh A patients (13.6 months) compared to Child-Pugh B patients (5.2 months), though the median time to progression (TTP) was similar (4.7 vs. 4.4 months, respectively) [25]. Though most patients received a starting dose of $800 \mathrm{mg} /$ day, the median dose in Child-Pugh A and B patients was $680 \mathrm{mg} /$ day and $721 \mathrm{mg} /$ day respectively, and the median duration of administration in the 2 groups was 13.7 weeks vs. 8.6 weeks, respectively [20].

Daniele et al. [26] evaluated sorafenib dosing and safety in the European subset of GIDEON, and reported that the majority of patients were started on $800 \mathrm{mg} /$ day, and that 800 and $400 \mathrm{mg} /$ day doses had similar adverse event profiles. However, the incidence of adverse events (all grades), drug-related adverse events, and serious adverse events was lower in patients treated with $800 \mathrm{mg} /$ day, with the exception of hand-foot skin reaction (HFSR), which was lower in the $400 \mathrm{mg} /$ day group. Subgroup analysis of the AP trial indicated that sorafenib treatment improved outcomes of advanced HCC patients regardless of baseline status [27].

The purpose of the present analysis was to investigate the safety of sorafenib in Chinese patients with unresectable HCC in real-world practice. This analysis also evaluated dosing in China, as well as the safety and efficacy in patients with different Child-Pugh class.

\section{Methods \\ Study design}

Data from the GIDEON Chinese subgroup were analyzed retrospectively. All included patients provided signed informed consent to participate in the GIDEON trial. The protocol and documentation were approved by the relevant institutional review boards, ethics committees and health authorities (Supplemental Data).

\section{Patients}

Out of a total of 345 Chinese patients with unresectable HCC identified in the GIDEON trial database, 331 were eligible for the current analysis. Eligible patients had a 
radiographic, histologic or cytologic diagnosis of unresectable HCC and had a life expectancy of at least 8 weeks [18]. Exclusion criteria were consistent with the locally approved sorafenib (Nexavar) product information [18]. All patient treatment decisions were determined solely at the treating physicians' discretion.

\section{Sorafenib treatment}

Indications for sorafenib were consistent with the local sorafenib package information. The dosage of sorafenib and duration of therapy were determined by treating physicians based on individual patient conditions. All included patients were treated with sorafenib at least once. The duration of observation was from the initiation of sorafenib to withdrawal from the study, final follow-up visit, or death. Patients were seen after initiating sorafenib, during therapy, and after completion of sorafenib therapy until withdrawal from the study, loss to followup, or death.

\section{Data collection}

Data collection for the GIDEON study has been previously described in detail [18-20]. Patient information was collected using both paper and electronic case report forms. At first visit, detailed patient demographics, baseline disease characteristics, previous therapies and sorafenib starting dose were recorded. At each physician visit, the date of the visit, $\mathrm{HCC}$ evaluation findings, and patient condition (as previously described), sorafenib therapy information (i.e., current dose, dose adjustments, and clinical benefit), adverse events and serious adverse events, and concomitant use of other drugs and therapies for HCC were recorded. All patient information was protected, and comparisons of patient data were done anonymously.

\section{Definitions of adverse events and serious adverse events}

For the purposes of this study, an adverse event was defined as any unfavorable or unintended symptom, sign, or disease (including abnormal laboratory results or state of mind) associated with the use of sorafenib. Such effects may or may not be intervention-related, dose-related, route-related, patient-related, or caused by an interaction with another drug, which must be specified in recording the adverse events. A serious adverse event was defined as an adverse event resulting in any of the following at any dose: death, life-threatening condition (i.e., high risk of death), hospitalization (except for observation, convalescence, or planned surgery), persistent or evident disability (impaired daily life), birth defects/congenital malformation, or other important medical events determined by clinicians.

\section{Study end points and safety and efficacy assessment}

The primary objective of the current analysis was to evaluate the safety of sorafenib in patients with unresectable HCC in routine clinical practice. All adverse events and serious adverse events occurring in all patients at any time, related or non-related to sorafenib therapy, were recorded, including date of presence or absence of adverse events, adverse event grade, relationship between adverse events and sorafenib (treatment-related adverse events), and measures taken to relieve the adverse events and related outcomes. The clinical characteristics of adverse events and serious adverse events, and the time from beginning sorafenib to occurrence of the adverse event were recorded. Adverse events were graded using the National Cancer Institute Common Terminology Criteria version 3.0 [28].

Dosage, duration of treatment, dose reductions/interruptions (plus reasoning), Child-Pugh class, ECOG-PS, and liver function were all documented at baseline and/ or follow-up. Efficacy end points included OS, progression-free survival (PFS), and time to progression (TTP) as defined by Response Evaluation Criteria In Solid Tumors [29].

\section{Statistical analysis}

All statistical analyses were performed with SAS software version 9.3 (SAS Institute Inc., Cary, NC). Adverse events analysis was conducted based on data from the safety population, and efficacy analysis was conducted on the intention-to-treat population. Patient demographic data and baseline characteristics were presented as mean and standard deviation for continuous variables,

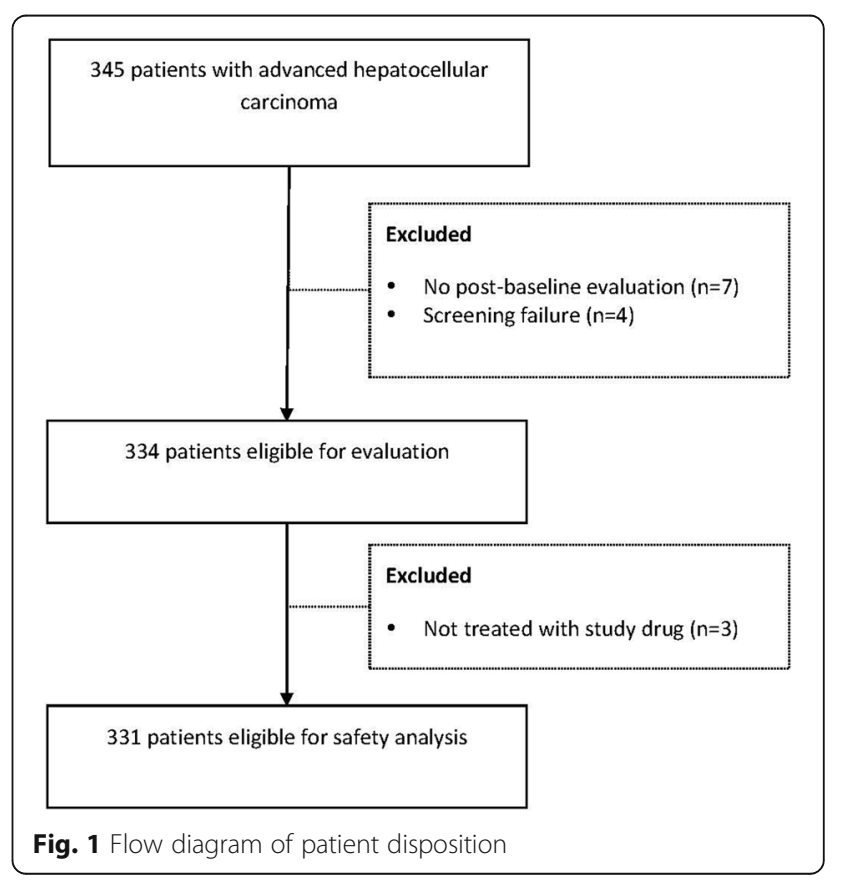


Table 1 Characteristics of the safety population $(N=331)$

\begin{tabular}{cl}
\hline Age (years) & $50.0 \pm 11.5$ \\
$<65$ & $297(90)$ \\
$65-75$ & $25(7.6)$ \\
$\geq 75$ & $7(2.1)$ \\
Missing & $2(0.6)$
\end{tabular}

Gender

Male

Female

Hepatitis B

Yes

No

Others $^{a}$

Portal vein thrombosis

Yes

No

Unknown

Number of liver lesions

$1-3$

$>3$

HCC confined to liver, yes

Vascular invasion, yes

Extrahepatic spread, yes

TNM stage

| or ||

III

IV

Missing or unknown

Barcelona Clinic Liver Cancer stage

$A$ or $B$

76 (23)

C or D

Not evaluable

Child-Pugh class

A

B or C

Not evaluable

Cancer of the Liver Italian Program score

0

1

2

3

4-6

Prior surgery

Prior locoregional therapy

None

Percutaneous ethanol injection only

305 (92)

$26(7.9)$

$296(89)$

$6(1.8)$

36 (11)

$98(30)$

$190(57)$

43 (13)

196 (59)

112 (34)

$142(43)$

74 (22)

$134(41)$

60 (18)

$129(39)$

72 (22)

70 (21)

$222(67)$

$33(10)$

$220(67)$

38 (12)

$73(22)$
Table 1 Characteristics of the safety population $(N=331)$

(Continued)

\begin{tabular}{ll}
\hline Radiofrequency ablation only & $16(4.8)$ \\
Transarterial chemoembolization only & $172(52)$ \\
Concomitant $^{\text {b }}$ & $50(15)$ \\
Others & $2(0.6)$ \\
\hline
\end{tabular}

Data are presented as mean \pm standard deviation or count (percentage).

Percentages are presented to 2 significant figs

HCC hepatocellular carcinoma, TNM tumor node metastasis

${ }^{a}$ Others include five hepatitis C, five alcohol use, 23 unknown, two other, and one other hepatobiliary disorders/findings

${ }^{b}$ Concomitant therapy refers to using at least two therapies for loco-regional

anti-cancer treatment at baseline

and by count and percentage for categorical variables. Safety data, including adverse events, serious adverse events, and death were presented as count and percentage according to Child-Pugh class at the start of therapy. Data regarding study drug administration were summarized as mean and standard deviation and median (range) for continuous data, and count and percentage for categorical data. The associations between baseline disease characteristics with OS, PFS, and TTP were assessed by the Kaplan-Meier method.

\section{Results}

\section{Patient characteristics at study entry}

Of the 345 Chinese patients in the database, 14 patients were excluded: seven did not attend any evaluations after enrollment, four failed screening, and three were not treated with the study drug. Thus, data of 331 eligible patients who met the inclusion criteria were included as the safety population (Fig. 1).

Patients demographic and baseline characteristics are summarized in Table 1 . The mean age of the safety population was $50.0 \pm 11.5$ years (range, 19-85 years), the majority of patients were male $(92 \%)$ and less than 65 years of age (90\%). Among all patients, 89\% had hepatitis B infection, 33\% had more than three liver lesions, $43 \%$ had HCC confined to the liver, $40.5 \%$ had extrahepatic HCC, $30 \%$ had portal vein thrombosis, and $22 \%$ had vascular invasion. Approximately $39 \%$ of patients were tumor-node-metastasis (TNM) stage at III, and $22 \%$ were stage IV. Based on the Barcelona Clinic Liver Cancer (BCLC) staging classification, 23\% were class A or B, and $67 \%$ of were class $C$ or D. Majority of patients, were Child-Pugh A (67\%) and 12\% were ChildPugh B or C. Prior to sorafenib treatment, 34\% patients received surgery and 52\% received TACE.

\section{Sorafenib administration}

Sorafenib administration of the overall study population is summarized in Table 2. Completed dosing data of 319 patients was available for review.. The median treatment duration was 22.3 weeks (range, $0.1-116.1$ weeks); $41 \%$ 
Table 2 Sorafenib administration in the safety population $(N=331)$

\begin{tabular}{ll}
\hline Duration of treatment & \\
Missing & $12(3.6)$ \\
$\leq 4$ weeks & $21(6.3)$ \\
$>4$ and $\leq 8$ weeks & $37(11)$ \\
$>8$ and $\leq 12$ weeks & $51(15)$ \\
$>12$ and $\leq 16$ weeks & $23(6.9)$ \\
$>16$ and $\leq 20$ weeks & $17(5.1)$ \\
$>20$ and $\leq 24$ weeks & $19(5.7)$ \\
$>24$ and $\leq 28$ weeks & $15(4.5)$ \\
$>28$ weeks & $136(41)$
\end{tabular}

Treatment duration (weeks) for available patients

\begin{tabular}{|c|c|}
\hline Number & 319 \\
\hline Mean \pm SD & $29.54 \pm 24.79$ \\
\hline Median (range) & $22.30(0.1$ to 116.1$)$ \\
\hline \multicolumn{2}{|l|}{ Average daily dose (mg) } \\
\hline Number & 204 \\
\hline Mean \pm S D & $776.2 \pm 83.6$ \\
\hline Median (range) & 800 (332 to 818 ) \\
\hline \multicolumn{2}{|l|}{ Total dose (mg) } \\
\hline Number & 204 \\
\hline Mean \pm SD & $133,512.3 \pm 115,060.0$ \\
\hline Median (range) & $198,350(800$ to 600,800 \\
\hline \multicolumn{2}{|l|}{ Dose intensity (\%) } \\
\hline Number & 204 \\
\hline Mean \pm SD & $96.5 \pm 11.8$ \\
\hline Median (range) & $100(26$ to 102$)$ \\
\hline \multicolumn{2}{|l|}{ Actual days on study drug (excluding off days) } \\
\hline Number & 204 \\
\hline Mean \pm SD & $171.4 \pm 146.3$ \\
\hline Median (range) & $127.5(1$ to 751$)$ \\
\hline \multicolumn{2}{|l|}{ Initial sorafenib dose level } \\
\hline Number & 331 \\
\hline $400 \mathrm{mg}$ & $4(1.2)$ \\
\hline $600 \mathrm{mg}$ & $1(0.3)$ \\
\hline 800 mg & $326(98)$ \\
\hline $\begin{array}{l}\text { Total subjects with dose interruptions ticked, } \\
\text { yes }\end{array}$ & $7(2.1)$ \\
\hline $\begin{array}{l}\text { Total subjects with dose modification ticked, } \\
\text { yes }\end{array}$ & $27(8.2)$ \\
\hline Total subjects with dose increase, yes & $13(3.9)$ \\
\hline Total subjects with dose reduction, yes & $21(6.3)$ \\
\hline
\end{tabular}

Data are presented as mean \pm standard deviation, median (range) or count (percentage). Percentages are presented to 2 significant figures

of patients had a treatment duration $>28$ weeks and only $6.3 \%$ had a treatment duration of $\leq 4$ weeks. Overall, $98 \%$ of patients received an initial dose of $800 \mathrm{mg} /$ day, and mean daily dose was $776.2 \pm 83.6 \mathrm{mg} /$ day (median daily dose $800 \mathrm{mg}$ ). Seven patients (2.1\%) had dose interruptions, and five of these (71\%) were due to adverse events. Twentyseven patients $(8.2 \%)$ required dose modification, and of these 16 (59\%) were modified due to adverse events. Thirteen patients (3.9\%) had a dose increase, and 21 (6.3\%) required a dose reduction (Additional file 1: Table S1).

\section{Safety assessment}

Safety data are reported in Tables 3, 4 and 5. Overall, $51 \%$ of patients experienced at least one treatmentemergent adverse event, and 6\% were grade 3-4 adverse events. Drug-related adverse events were experienced by $29 \%$ of patients, and $3.6 \%$ had grade 3 or 4 events. Overall, $23 \%$ of patients $(n=77)$ experienced serious adverse events, and only one ( $0.3 \%)$ was drug-related (Table 3$)$.

The incidence of adverse events and serious adverse events were broadly comparable between Child Pugh A and $\mathrm{B}$ patients. The majority of drug-related adverse events were grade 1 or 2 in Child-Pugh A and B patients, though the frequency of drug-related adverse events was greater in Child-Pugh B (35\%) compared with Child-Pugh A (27\%) patients (Table 3). The incidences of treatment-emergent adverse events by worst grade and Child-Pugh score at start of therapy are summarized in Additional file 1: Table S2.

The most frequent drug-related adverse events were comparable across Child-Pugh subgroups, and were consistent with those of the overall population (Table 4). Dermatological, HFSR, gastrointestinal, and diarrhea were the most frequently observed in both Child-Pugh A and B patients, with gastrointestinal adverse events slightly more frequent in Child-Pugh B than A patients (17\% vs $10 \%)$.

The time to onset of all adverse events was within 30 days of starting sorafenib, then declined markedly (Fig. 2). However, the time to onset for specific adverse events varied (Table 5). Of 39 patients with diarrhea, 35 were drugrelated. Onset of diarrhea most frequently occurred within 30 days, and after 90 days. Of 64 patients with HFSR, 63 were drug-related and the onset was typically within 30 days. Liver dysfunction occurred most frequently after 90 days with no drug-related cases reported.

\section{Efficacy (intention-to-treat population)}

Overall 378 patients were in interntion-to-treat population, there were 166 mortality events,222 progression events as defined by the Response Evaluation Criteria in Solid Tumors criteria. The median OS was 322 days (95\% confidence interval [CI]: 270-382 days), the median PFS was 186 days (95\% CI: 155-216 days), and median TTP was 274 days (95\% CI: 209-463 days). The median OS was longer for Child-Pugh A patients 
Table 3 Treatment-emergent adverse events by Child-Pugh class at start of therapy in the safety population

\begin{tabular}{|c|c|c|c|c|c|}
\hline Adverse events & $\begin{array}{l}\text { Total } \\
(N=331)\end{array}$ & $\begin{array}{l}\text { Child-Pugh A } \\
(n=246)\end{array}$ & $\begin{array}{l}\text { Child-Pugh B } \\
(n=48)\end{array}$ & $\begin{array}{l}\text { Child-Pugh C } \\
(n=2)\end{array}$ & $\begin{array}{l}\text { Not evaluable } \\
(n=35)\end{array}$ \\
\hline Treatment-emergent AE (all grades including deaths not documented as AE) & $233(70)$ & $175(71)$ & $38(79)$ & $0(0)$ & $20(57)$ \\
\hline AEs resulting in permanent discontinuation & $29(8.8)$ & $22(8.9)$ & $5(10)$ & $0(0)$ & $2(5.7)$ \\
\hline Treatment-emergent AE (all grades) & $167(51)$ & $124(50)$ & $27(56)$ & $0(0)$ & $16(46)$ \\
\hline Grade 3-4 & $20(6.0)$ & $14(5.7)$ & $4(8.3)$ & $0(0)$ & $20(6.0)$ \\
\hline Grade 5 & $73(22)$ & $55(22)$ & $11(23)$ & $0(0)$ & $7(20)$ \\
\hline Drug-related AE (all grades) & $95(29)$ & $67(27)$ & $17(35)$ & $0(0)$ & $11(31)$ \\
\hline Grade 3-4 & $12(3.6)$ & $9(3.7)$ & $2(4.2)$ & $0(0)$ & $1(2.9)$ \\
\hline Grade 5 & $0(0)$ & $0(0)$ & $0(0)$ & $0(0)$ & $0(0)$ \\
\hline Serious AE (all grades) & $77(23)$ & $58(24)$ & $12(25)$ & $0(0)$ & $7(20)$ \\
\hline Drug-related (all grades) & $1(0.3)$ & $0(0)$ & $1(2.1)$ & $0(0)$ & $0(0)$ \\
\hline All deaths & $166(50)$ & $127(52)$ & $27(56)$ & $0(0)$ & $12(34)$ \\
\hline Treatment-emergent death & $73(22)$ & $55(22)$ & $11(23)$ & $0(0)$ & $7(20)$ \\
\hline
\end{tabular}

Data are presented as count (percentage). Percentages are presented to 2 significant figs

$A E$ adverse event, $S A E$ serious adverse event

Treatment-emergent deaths: death while on sorafenib and up to 30 days after last dose collected from all available sources

All deaths: all deaths from all sources where death information was collected including follow-up information

compared to B (322 vs. 240 days), while the PFS time (183 vs. 208 days), and median TTP (214 days vs. not reached) were shorter for Child-Pugh A patients compared to Child-Pugh B patients (Fig. 3).

\section{Discussion}

The majority of this Chinese subgroup was male (92\%), more than 65 years old (90\%), and had hepatitis B (89\%).

Table 4 Incidence of treatment-emergent drug-related adverse events by worst grade and Child-Pugh class at start of therapy

\begin{tabular}{llll}
\hline $\begin{array}{l}\text { Drug-related adverse } \\
\text { events }\end{array}$ & $\begin{array}{l}\text { Child-Pugh A } \\
(n=246)\end{array}$ & $\begin{array}{l}\text { Child-Pugh B } \\
(n=48)\end{array}$ & $\begin{array}{l}\text { Child-Pugh C } \\
(n=2)\end{array}$ \\
\hline Any adverse event & $67(27)$ & $17(35)$ & $0(0)$ \\
Cardiac, general & $8(3.3)$ & $1(2.1)$ & $0(0)$ \\
Hypertension & $7(2.8)$ & $1(2.1)$ & $0(0)$ \\
Hypotension & $2(0.8)$ & $0(0)$ & $0(0)$ \\
Constitutional symptoms & $2(0.8)$ & $0(0)$ & $0(0)$ \\
Fatigue & $1(0.4)$ & $0(0)$ & $0(0)$ \\
Fever & $1(0.4)$ & $0(0)$ & $0(0)$ \\
Dermatology/skin & $58(24)$ & $12(25)$ & $0(0)$ \\
Alopecia & $6(2.4)$ & $2(4.2)$ & $0(0)$ \\
Hand-foot skin reaction & $51(21)$ & $7(15)$ & $0(0)$ \\
Rash/desquamation & $8(3.3)$ & $3(6.3)$ & $0(0)$ \\
Ulceration & $0(0)$ & $0(0)$ & $0(0)$ \\
Gastrointestinal & $25(10)$ & $8(17)$ & $0(0)$ \\
Diarrhea & $25(10)$ & $7(15)$ & $0(0)$ \\
Nausea & $0(0)$ & $1(2.1)$ & $0(0)$ \\
\hline
\end{tabular}

Data are presented as count (percentage). Percentages are presented to 2 significant figures
This population consisted of $67 \%$ Child-Pugh A patients, and $67 \%$ were BCLC stage C or D compared to the overall GIDEON safety population, where $61 \%$ of patients were Child-Pugh A and $21 \%$ were Child-Pugh B. In reallife clinical practice, over $98 \%$ of patients received the recommended initial sorafenib dose of $800 \mathrm{mg} /$ day, with a median duration of treatment of 22.3 weeks (range, 0.1-116.1 months). In comparison to the overall GIDEON safety population,, where only $72 \%$ of Child-Pugh A and 70\% Child-Pugh B patients received an initial sorafenib dose of $800 \mathrm{mg}$ [30].

\section{Adverse events}

About $50 \%$ of patients experienced at least one adverse event, with approximately $28 \%$ experiencing a drugrelated adverse event. Majority of the drug-related adverse events were grade 1 or 2 , which is consistent with what was reported in the GIDEON and Asia-Pacific phase III trials $[13,14]$. The results are different from the SHARP trial in which $98 \%$ of sorafenib-treated patients had an adverse event, and $80 \%$ had a drug-related adverse event [23]. However, the incidence of serious adverse events was lower (23\%) in the GIDEON Chinese subgroup compared with the serious adverse events incidence in the SHARP study (52\%) [23].

In this subgroup, the most common drug-related adverse events were dermatology/skin, HFSR, gastrointestinal, and diarrhea which is similar to previous trials $[14,19,23]$. However, a very low percentage of patients reported having fatigue $(0.4 \%)$ or rash $(3.3 \%$ and $6.3 \%$ for patients with Child-Pugh A and Child-Pugh B, respectively), which differs from SHARP [23], AP [14], and GIDEON [19], where fatigue and rash/desquamation were some of the most 
Table 5 Onset time for drug-related adverse events: diarrhea, hand-foot skin reaction, and liver dysfunction

\begin{tabular}{lllll}
\hline Time of onset & Total & Child-Pugh A & Child-Pugh B & Not evaluable \\
\hline Diarrhea & $35 / 39$ & $25 / 28$ & $7 / 8$ & $3 / 3$ \\
$0-30$ days & $12 / 13$ & $8 / 8$ & $1 / 1$ & $0 / 0$ \\
$31-60$ days & $4 / 4$ & $3 / 3$ & $0 / 0$ & $1 / 0$ \\
$61-90$ days & $5 / 5$ & $4 / 4$ & $2 / 2$ & $2 / 2$ \\
$>90$ days & $14 / 17$ & $10 / 13$ & $7 / 8$ & $5 / 5$ \\
Hand-foot skin reaction & $63 / 64$ & $51 / 51$ & $5 / 6$ & $3 / 3$ \\
$0-30$ days & $37 / 38$ & $29 / 29$ & $1 / 1$ & $1 / 1$ \\
$31-60$ days & $11 / 12$ & $9 / 10$ & $0 / 0$ & $0 / 0$ \\
$61-90$ days & $5 / 5$ & $5 / 5$ & $1 / 1$ & $1 / 1$ \\
$>90$ days & $10 / 9$ & $8 / 7$ & $0 / 9$ & $0 / 7$ \\
Liver dysfunction & $0 / 46$ & $0 / 30$ & $0 / 2$ & $0 / 2$ \\
$0-30$ days & $0 / 6$ & $0 / 2$ & $0 / 1$ & $0 / 0$ \\
$31-60$ days & $0 / 2$ & $0 / 1$ & $0 / 1$ & $0 / 1$ \\
61-90 days & $0 / 10$ days & $0 / 8$ & $0 / 5$ & \\
\hline
\end{tabular}

Data are presented as represented as number of drug-related adverse events / number of adverse events

commonly reported drug-related adverse events. In the AP study, the most common grade 3 and 4 drug-related adverse events in sorafenib-treated patients were HFSR, diarrhea, and fatigue, and fatigue was the most common in patients receiving placebo [27]. Al-Rajabi et al. [24] reported nausea in $70.8 \%$ of all patients, and diarrhea in $57.5 \%$, and the percentages were similar between ChildPugh A and B patients. In general, gastrointestinal distress (diarrhea) is a common adverse event associated with sorafenib, whether it is used for HCC or advanced renal cell carcinoma [31, 32]. Other studies of GIDEON Chinese subset have shown sorafenib is associated with adverse events regardless of prior surgery, the presence of portal vein tumor thrombosis [22], or with prior or concomitant TACE [21].

\section{Influence of child-Pugh class on safety}

Overall, adverse events were comparable between ChildPugh A and B patients, including the most frequently occurring drug-related adverse events. However, the Chinese subgroup differed from the overall global GIDEON cohort with regard to drug-related adverse events with more events occurring in Child-Pugh B (35\%) vs. Child-Pugh A (27\%) patients.. Additionally, the percentage of Chinese patients experiencing drug-related adverse events was much lower than reported for the overall GIDEON cohort in the second interim analysis (63\% for Child-Pugh B; 67\% for Child Pugh A) [20]. Toxicity in terms of all-grade adverse events was lower in Child-Pugh A (50\%) and B (56\%) patients. In addition, there were no differences between Child-Pugh A and B patients with respect to the percentage of deaths and treatment-emergent deaths. A serious drug-related adverse event was only reported in one Child-Pugh B patient (0.3\%), and none were reported in Child-Pugh A patients. The two Child-Pugh $C$ patients had no adverse events, serious adverse events, or death; however, this number is too small to make meaningful comparisons.

Differences in patient baseline clinical characteristics, in initial sorafenib dose and administration, and in the concomitant locoregional therapy may have contributed to differences between this study and others. In the present study, initial sorafenib dose of $800 \mathrm{mg}$ per day was the most common dosage, which is consistent with most studies. Adverse events were the main reason for dose interruptions and drug discontinuation in the present study, and in other studies. Reasons for dose modification included adverse events, disease progression, and concomitant treatments/procedures (Additional file 1: Table S1). Our results suggest that a longer duration of sorafenib treatment with the recommended daily dose of $800 \mathrm{mg}$ may contribute to greater clinical benefit in terms of survival with an acceptable safety profile.

\section{Influence of child-Pugh class on efficacy}

In the present study the overall median OS was 322 days (10.7 months), compared with the median OS recorded in the Asia-pacific study (6.5 months for total AP population) [27]. It should be noted, however, that although our observed median OS was longer, in our population $22 \%$ and $41 \%$ of the patients had tumor burden, vascular invasion and extra-hepatic spread, respectively; as compared 


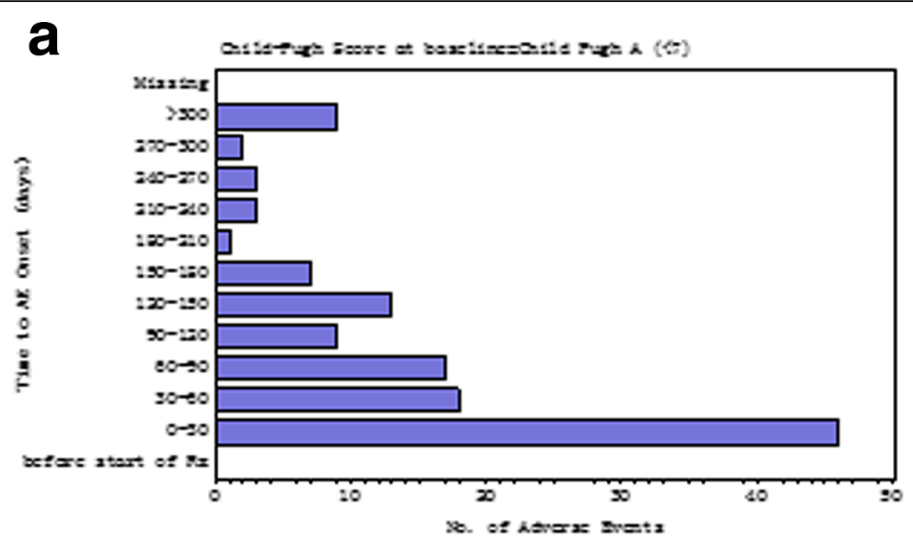

b
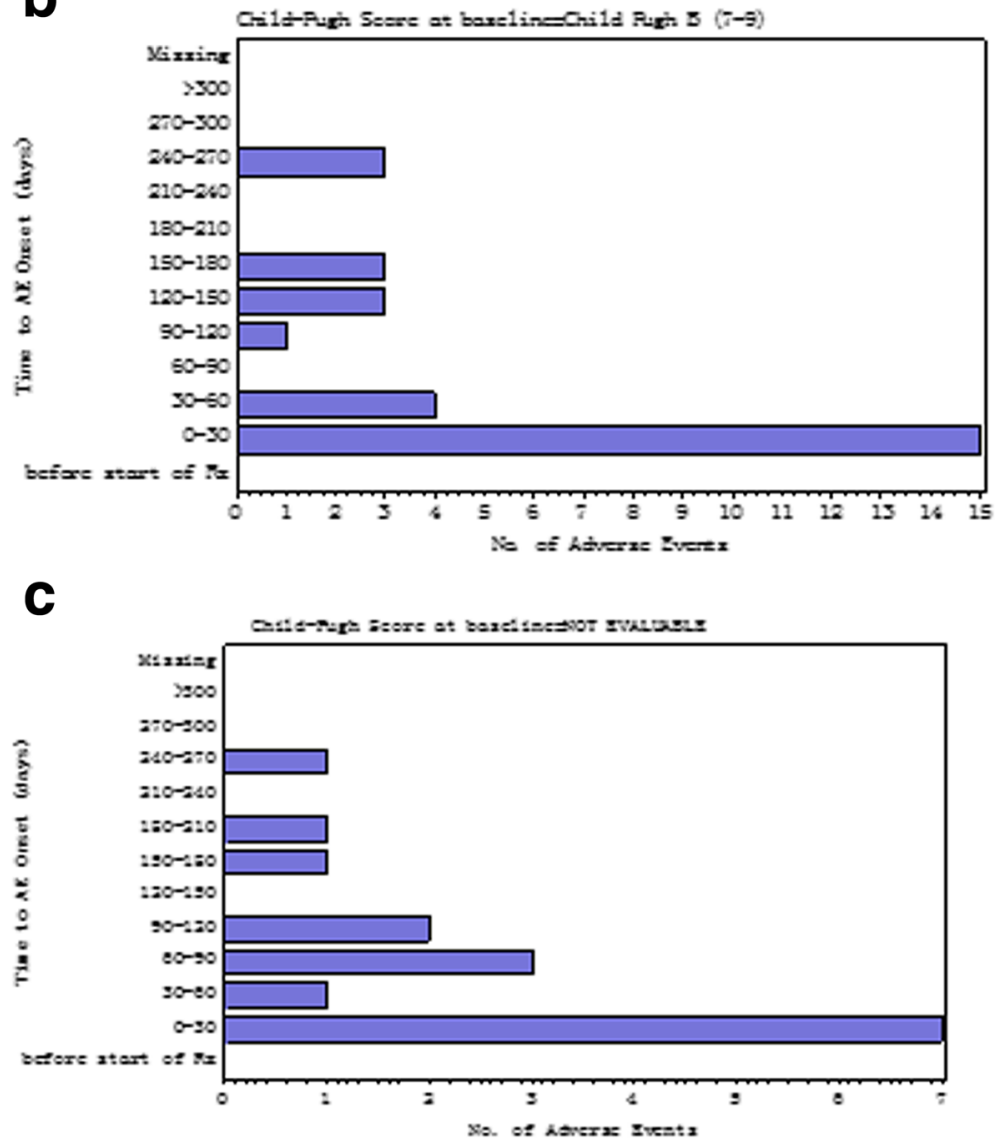

Fig. 2 Time to adverse events onset after sorafenib administration in the safety population. a Child-Pugh class A $(n=246)$. b Child-Pugh class B $(n=38)$. c Child-Pugh class not evaluable $(n=35)$. Child-Pugh classes are those at baseline, minimum grade 1. AE: adverse events

to $79 \%$ of the patients had either or both tumor burden in the Asian-pacific study. These comparisons suggest that patients with less advanced disease at the start of therapy may experience a greater survival benefit.

Final analysis of the GIDEON data showed the median OS was greater in Child-Pugh A patients (13.6 months, 95\% CI: $12.8-14.7$ months) than in B patients (5.2 months, 95\% CI: 4.6-6.3 months) [25]. Consistent with the findings of a recent study by Al-Rajabi et al. [24], our population, with $11.5 \%$ Child-Pugh B patients, had a difference in median OS between Child-Pugh A and B (10.7 vs. 8 months, respectively). In the second interim analysis of the GIDEON data, most patients received the approved sorafenib dose of $800 \mathrm{mg} /$ day, but the median duration of therapy was shorter in Child-Pugh B patients [20]. A recent European subgroup analysis of GIDEON data suggested that a higher starting dose may result in longer OS [26]. Liu et al. [21] reported that the Chinese 


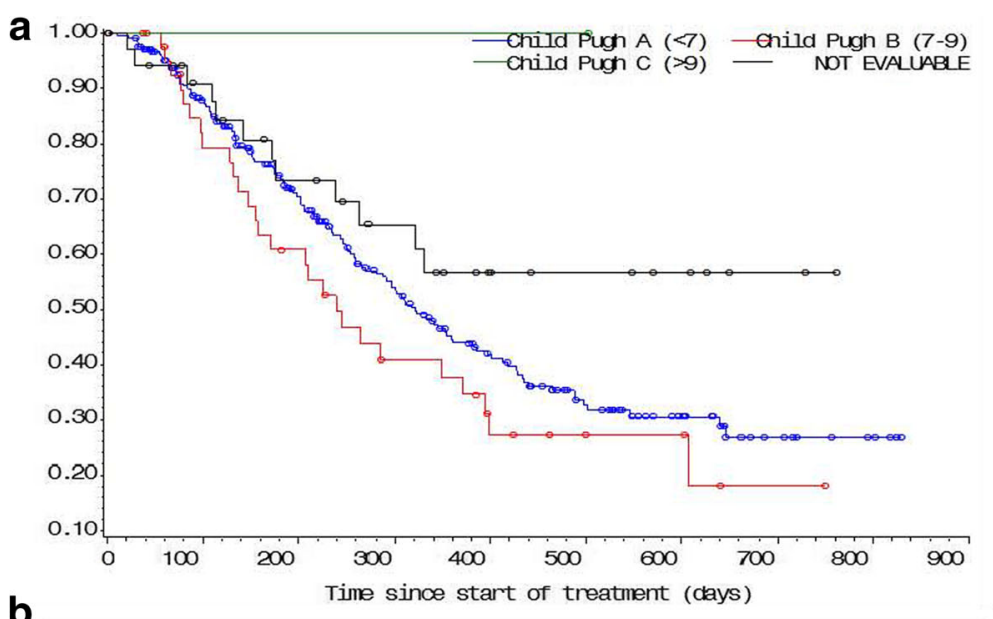

b

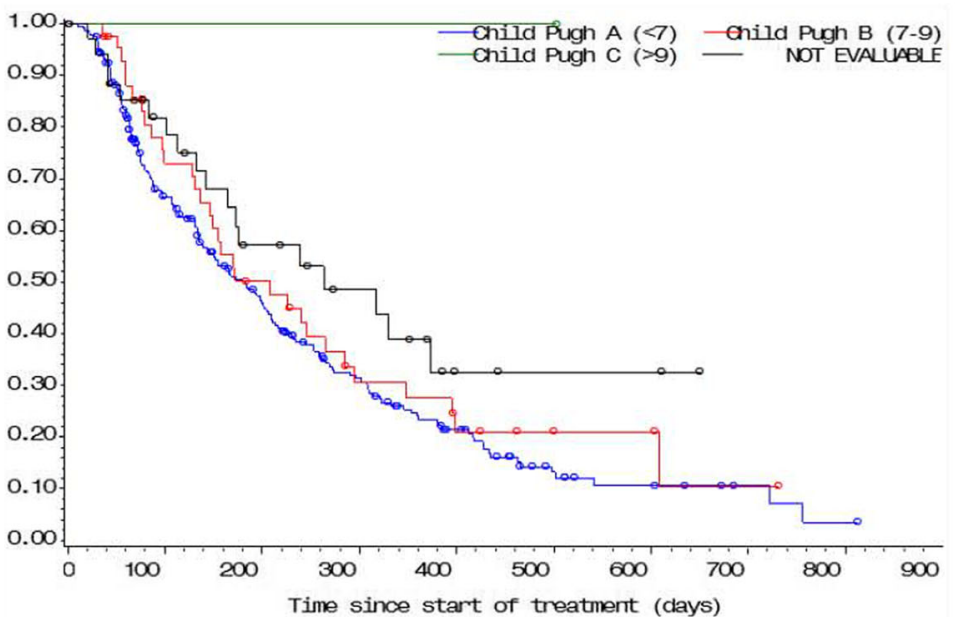

C

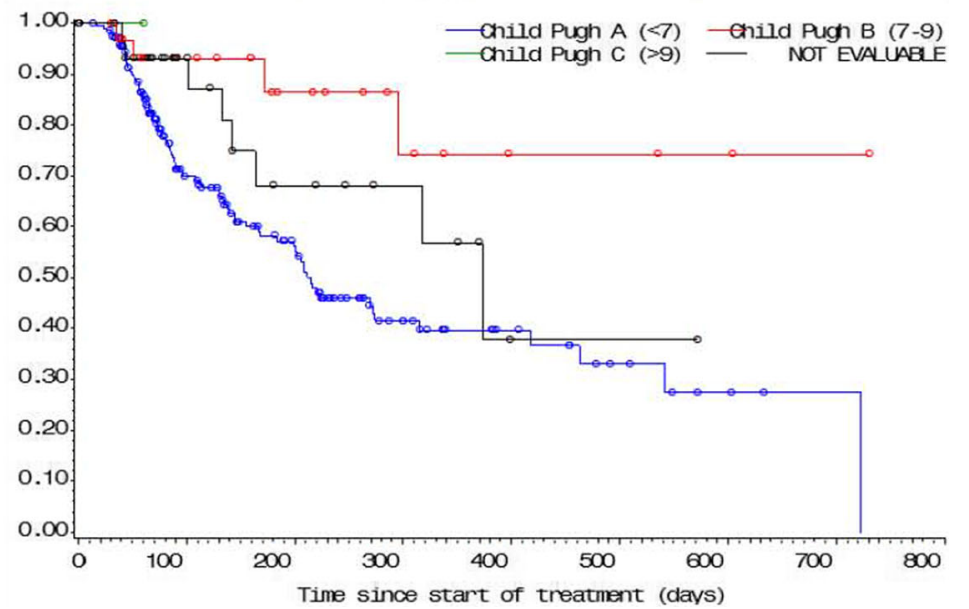

Fig. 3 Kaplan-Meier curve survival for patients with unresectable HCC based on Child-Pugh score in the intention-to-treat population. a Overall survival (OS); (b) progression-free survival (PFS); (c) time-to-progression for Child-Pugh A versus Child-Pugh B, Child-Pugh C as defined by Response Evaluation Criteria in Solid Tumors criteria

subpopulation had more advanced HCC at study entry than the overall GIDEON population (BCLC C/D, $67 \%$ vs. $58 \%$; TNM III/IV, 77\% vs. 72\%), but better underlying liver function (Child-Pugh $\mathrm{B} / \mathrm{C}, 16 \%$ vs. $58 \%$ ), and Chinese patients with the best liver function (Child-Pugh A) had a longer OS than GIDEON patients as a whole (13.6 months vs. 10.7 months), while those with worse liver function (ChildPugh B) had a shorter OS (5.0 months vs. 8.0 months). 


\section{Limitations}

This study has certain limitations, including the retrospective analysis of data which limits evaluation of causality. The descriptive results should be interpreted with caution as statistical analysis for significance was not performed. Data were collected from multiple tertiary care centers in China, and the findings may not translate fully to daily practice in primary care settings. Whether patients were given continuous or interrupted sorafenib dosing was not studied. In addition, only $1.2 \%$ of patients received an initial dose of $400 \mathrm{mg}$, and thus the safety and efficacy of this dose could not be studied. Furthermore, only $12 \%$ of patients were Child-Pugh B or C, and among them there were only two evaluable ChildPugh C patients. Similarly, the percentages of patients receiving a $400 \mathrm{mg}(1.2 \%)$ and $600 \mathrm{mg}$ (0.3\%) starting dose were too small to allow a meaningful comparison. Lastly, we did not collect data or study the influence of hepatitis B viral load.

\section{Conclusions}

This study has shown that sorafenib has acceptable efficacy and safety in Chinese Child-Pugh A and B patients with unresectable $\mathrm{HCC}$ using the recommended dosage of $800 \mathrm{mg} /$ day, and that safety and the occurrence of adverse events are not affected by liver function. Different than seen in other phase III sorafenib trials, gastrointestinal adverse events were the most common drug-related adverse events in Chinese patients with unresectable HCC administered sorafenib. Taken together, these results suggest that sorafenib treatment is appropriate for Chinese patients with unresectable HCC.

\section{Additional file}

Additional file 1: Table S1. Reasons for dose interruptions or dose modifications. Table S2. Incidences of treatment-emergent adverse events by worst grade and Child-Pugh score at start of therapy are summarized. (DOC $63 \mathrm{~kb}$ )

\section{Abbreviations}

AE: adverse event; BCLC: Barcelona Clinic Liver Cancer; Cl: confidence interval; CT: computed tomography; ECOG-PS: Eastern Cooperative Oncology Group performance status; GIDEON: Global Investigation of therapeutic DEcisions in hepatocellular carcinoma and Of its treatment with sorafeNib; HCC: hepatocellular carcinoma; HFSR: hand-foot skin reaction; OS: overall survival; PFS: progression-free survival; SAE: serious adverse event; SD: standard deviation; SHARP: Sorafenib Hepatocellular Carcinoma Assessment Randomised Protocol trial; TACE: transarterial chemoembolization; TNM: tumor-node-metastasis; TTP: time to progression

\section{Acknowledgements}

None.

\section{Funding}

This trial was funded by Bayer HealthCare Pharmaceutical Company, Ltd., China. Bayer played a role in data collection.

\section{Availability of data and materials}

The datasets used and/or analyzed during the current study are available from the corresponding author on reasonable request.

\section{Authors' contributions}

Conception and study design: SLY, JMY, PB, SJZ, XPC, FYL, LML, JZ, KFD, CYH, GLS, QX, YJC, JJY, XXD, YPL, YFY, ZRF. Analysis of data: SLY, KN, ZGL. Data interpretation: SLY. In drafting the manuscript: SLY, KN, ZGL. Critically revising the manuscript: SLY. All authors have read and approved the final version of this manuscript.

\section{Ethics approval and consent to participate}

This study was approved by the ethics committee of Zhongshan Hospital Fudan University (certification number: 2009-15). Written informed consent was obtained from all the patients involved.

The GIDEON study was an international prospective, open-label, multi-centered, non-interventional study of patients with unresectable HCC who had indications for systemic therapy and received sorafenib. All patients met the inclusion and exclusion criteria, were treated with sorafenib at least once. The duration of observation was from the initiation of sorafenib therapy to withdrawal from the study, last follow-up, or death. The GIDEON study protocol [NCT00812175] is available at: https://www.clinicaltrials.gov/ct2/show/NCT00812175).

\section{Consent for publication}

Not applicable.

\section{Competing interests}

This trial was financially supported by Bayer HealthCare Pharmaceutical Company, Ltd., China.

\section{Publisher's Note}

Springer Nature remains neutral with regard to jurisdictional claims in published maps and institutional affiliations.

\section{Author details}

${ }^{1}$ Liver Cancer Institute, Zhongshan Hospital, Fudan University, 136 Yixueyuan Rd, Shanghai 200032, China. ${ }^{2}$ Department of Special Treatment, Eastern Hepatobiliary Surgery Hospital, Shanghai, China. ${ }^{3}$ Institute of Hepatobiliary Surgery, Southwest Hospital, Third Military Medical University, Chongqing, China. ${ }^{4}$ Department of General Surgery, The First Affiliated Hospital of Zhengzhou University, Zhengzhou, China. ${ }^{5}$ Department of Surgery, Tongji Medical College, Huazhong University of Science and Technology, Wuhan, China. ${ }^{6}$ Department of Interventional Radiology, Chinese PLA General Hospital, Beijing, China. ${ }^{7}$ Shanghai Cancer Center, Fudan University, Shanghai, China. ${ }^{8}$ Department of Hepatobiliary Surgery, Nanfang Hospital of Southern Medical University, Guangzhou, China. ${ }^{9}$ Department of Hepatobiliary Surgery, Xijing Hospital, Xi'an, China. ${ }^{10}$ Department of Hepato-Pancreato-Biliary Surgery, Beijing Cancer Hospital, Peking University, Beijing, China.

${ }^{11}$ Department of Radiology, Zhejiang Cancer Hospital, Hangzhou, China.

${ }^{12}$ Department of Liver Surgery, Renji Hospital, Shanghai Jiaotong University School of Medicine, Shanghai, China. ${ }^{13}$ Department of Hepatobiliary Surgery, Second Affiliated Hospital of Sun Yat-Sen University, Guangzhou, China.

${ }^{14}$ Department of Nuclear Medicine, Changhai Hospital, Second Military Medical University, Shanghai, China. ${ }^{15}$ Department of General Surgery, Ruijin Hospital, Shanghai Jiaotong University School of Medicine, Shanghai, China. ${ }^{16}$ Department of Medical Oncology, the First Hospital of China Medical University, Shenyang, China. ${ }^{17}$ Department of Hepatobiliary, Cancer Center, Sun Yat-sen University, Guangzhou, China. ${ }^{18}$ Department of Liver Transplantation, Shanghai Changzheng Hospital, Shanghai, China. ${ }^{19}$ Bayer Healthcare Pharmaceuticals, Whippany, NJ, USA. ${ }^{20}$ Bayer Healthcare Company Ltd., Beijing, China.

Received: 9 November 2016 Accepted: 19 February 2018

Published online: 02 March 2018

\section{References}

1. Ferlay J, Soerjomataram I, Dikshit R, Eser S, Mathers C, Rebelo M, Parkin DM, Forman D, Bray F. Cancer incidence and mortality worldwide: sources, methods and major patterns in GLOBOCAN 2012. Int J Cancer. 2015;136:E359-86. 
2. Torre LA, Bray F, Siegel RL, Ferlay J, Lortet-Tieulent J, Jemal A. Global cancer statistics, 2012. CA Cancer J Clin. 2015;65:87-108.

3. Makuchi M, Kokudo N. Clinical practice guidelines for hepatocellular carcinoma: the first evidence-based guidelines from Japan. World J Gastroenterol. 2006;12:828-9.

4. Qin S, Primary Liver Cancer Diagnosis and Treatment Expert Panel of the Chinese Ministry of Health. Guidelines on the diagnosis and treatment of primary liver cancer (2011 edition). Chin Clin Oncol. 2012;1:10.

5. Hung $\mathrm{H}$. Treatment modalities for hepatocellular carcinoma. Curr Cancer Drug Targets. 2005;5:131-8.

6. Bruix J, Sherman M. Management of hepatocellular carcinoma. Hepatology. 2005:42:1208-36.

7. Chang YS, Adnane J, Trail PA, Levy J, Henderson A, Xue D, Bortolon E, Ichetovkin M, Chen C, McNabola A, et al. Sorafenib (BAY 43-9006) inhibits tumor growth and vascularization and induces tumor apoptosis and hypoxia in RCC xenograft models. Cancer Chemother Pharmacol. 2007:59:561-74.

8. Raut CP, Boucher Y, Duda DG, Morgan JA, Quek R, Ancukiewicz M, Lahdenranta J, Eder JP, Demetri GD, Jain RK. Effects of sorafenib on intratumoral interstitial fluid pressure and circulating biomarkers in patients with refractory sarcomas (NCI protocol 6948). PLoS One. 2012;7:e26331.

9. Cabrera R, Pannu DS, Caridi J, Firpi RJ, Soldevila-Pico C, Morelli G, Clark V, Suman A, George TJ Jr, Nelson DR. The combination of sorafenib with transarterial chemoembolisation for hepatocellular carcinoma. Aliment Pharmacol Ther. 2011;34:205-13.

10. Pawlik TM, Reyes DK, Cosgrove D, Kamel IR, Bhagat N, Geschwind JF. Phase Il trial of sorafenib combined with concurrent transarterial. chemoembolization with drug-eluting beads for hepatocellular carcinoma. J Clin Oncol. 2011;29:3960-7.

11. Sieghart W, Pinter M, Reisegger M, Müller C, Ba-Ssalamah A, Lammer J, Peck-Radosavljevic M. Conventional transarterial chemoembolisation in combination with sorafenib for patients with hepatocellular carcinoma: a pilot study. Eur Radiol. 2012;22:1214-23.

12. Sansonno D, Lauletta G, Russi S, Conteduca V, Sansonno L, Dammacco F. Transarterial chemoembolization plus sorafenib: a sequential therapeutic scheme for HCV-related intermediate-stage hepatocellular carcinoma: a randomized clinical trial. Oncologist. 2012;17:359-66.

13. Llovet JM, Ricci S, Mazzaferro V, Hilgard P, Gane E, Blanc JF, de Oliveira AC, Santoro A, Raoul JL, Forner A, et al. Sorafenib in advanced hepatocellular carcinoma. N Engl J Med. 2008;359:378-90.

14. Cheng AL, Kang YK, Chen Z, Tsao CJ, Qin S, Kim JS, Luo R, Feng J, Ye S, Yang TS, et al. Efficacy and safety of sorafenib in patients in the Asia-Pacific region with advanced hepatocellular carcinoma: a phase III randomised, double-blind placebo-controlled trial. Lancet Oncol. 2009;10:25-34.

15. Kudo M, Imanaka K, Chida N, Nakachi K, Tak WY, Takayama T, Yoon JH, Hori T, Kumada H, Hayashi N, et al. Phase III study of sorafenib after transarterial chemoembolisation in Japanese and Korean patients with unresectable hepatocellular carcinoma. Eur J Cancer. 2011:47:2117-27.

16. Park JW, Koh YH, Kim HB, Kim HY, An S, Choi Jl, Woo SM, Nam BH. Phase II study of concurrent transarterial chemoembolization and sorafenib in patients with unresectable hepatocellular carcinoma. Hepatol. 2012;56:1336-42.

17. Han G, Yang J, Shao G, Teng G, Wang M, Yang J, Liu Z, Feng G, Yang R, Lu $L$, et al. Sorafenib in combination with transarterial chemoembolization in Chinese patients with hepatocellular carcinoma: a subgroup interim analysis of the START trial. Future Oncol. 2013;9:403-10.

18. Lencioni R, Marrero J, Venook A, Ye SL, Kudo M. Design and rationale for the non-interventional global investigation of therapeutic decisions in hepatocellular carcinoma and of its treatment with sorafenib (GIDEON) study. Int J Clin Pract. 2010;64:1034-41.

19. Lencioni R, Kudo M, Ye SL, Bronowicki JP, Chen XP, Dagher L, Furuse J, Geschwind JF, Ladrón de Guevara L, Papandreou C, et al. First interim analysis of the GIDEON (global investigation of therapeutic DEcisions in hepatocellular carcinoma and of its treatment with sorafeNib) noninterventional study. Int J Clin Pract. 2012;66:675-83.

20. Lencioni R, Kudo M, Ye SL, Bronowicki JP, Chen XP, Dagher L, Furuse J, Geschwind JF, de Guevara LL, Papandreou C, et al. GIDEON (global investigation of therapeutic DEcisions in hepatocellular carcinoma and of its treatment with sorafeNib): second interim analysis. Int J Clin Pract. 2014;68:609-17.

21. Liu F, Meng Z, Shao G, Wang J, Wang Z, Yang J, Yip CS, He D. Patterns of sorafenib and TACE treatment of unresectable hepatocellular carcinoma in a Chinese population: subgroup analysis of the GIDEON study. Mol Biol Rep. 2017:44:149-58

22. Ye SL, Chen X, Yang J, Bie P, Zhang S, Liu F, Liu L, Zhou J, Dou K, Yip CS, Yang X. Evaluation of sorafenib in Chinese unresectable hepatocellular carcinoma patients with prior surgery and portal vein tumor thrombosis: a subset analysis of GIDEON study data. Tumour Biol 2017:39(3): 1010428317695030

23. Bruix J, Raoul JL, Sherman M, Mazzaferro V, Bolondi L, Craxi A, Galle PR, Santoro A, Beaugrand M, Sangiovanni A, et al. Efficacy and safety of sorafenib in patients with advanced hepatocellular carcinoma: subanalyses of a phase III trial. J Hepatol. 2012;57:821-9.

24. Al-Rajabi R, Patel S, Ketchum NS, Jaime NA, Lu TW, Pollock BH, Mahalingam D. Comparative dosing and efficacy of sorafenib in hepatocellular cancer patients with varying liver dysfunction. J Gastrointest Oncol. 2015;6:259-67.

25. Marrero JA, Lencioni R, Ye SL, Masatoshi K, Bronowicki JP, Chen XP, Dagher L, Furuse J, Geshwind JF, de Guevara LL, et al. Final analysis of GIDEON (Global investigation of therapeutic decisions in hepatocellular carcinoma (HCC) and of its treatment with Sorafenib (Sor) in >3000 sor-treated patients (pts): clinical findings in pts with liver dysfunction. J Clin Oncol. 2013;31(15_suppl) 4126 [Abstract]. https://doi.org/10.1200/jco.2013.31.

26. Daniele B. Impact of sorafenib dosing on outcome from the European patient subset of the GIDEON study. Future Oncol. 2015;9:1-10. [Epub ahead of print]

27. Cheng AL, Guan Z, Chen Z, Tsao CJ, Qin S, Kim JS, Yang TS, Tak WY, Pan H, Yu S, et al. Efficacy and safety of sorafenib in patients with advanced hepatocellular carcinoma according to baseline status: subset analyses of the phase III Sorafenib Asia-Pacific trial. Eur J Cancer. 2012;48:1452-65.

28. Trotti A, Colevas AD, Setser A, Rusch V, Jaques D, Budach V, Langer C, Murphy B, Cumberlin R, Coleman CN, et al. CTCAE v3.0: development of a comprehensive grading system for the adverse effects of cancer treatment. Semin Radiat Oncol. 2003;13:176-81.

29. Eisenhauer EA, Therasse P, Bogaerts J, Schwartz LH, Sargent D, Ford R, Dancey J, Arbuck S, Gwyther S, Mooney M, et al. New response evaluation criteria in solid tumours: revised RECIST guideline (version 1.1). Eur I Cancer. 2009;45:228-47.

30. Marrero JA, Kudo M, Venook AP, Ye SL, Bronowicki JP, Chen XP, Dagher L, Furuse J, Geschwind JH, de Guevara LL, Papandreou C, Takayama T, Sanyal AJ, Yoon SK, Nakajima K, Lehr R, Heldner S, Lencioni R. Observational registry of sorafenib use in clinical practice across child-Pugh subgroups: the GIDEON study. J Hepatol. 2016;65:1140-7.

31. Santoni M, Conti A, De Giorgi U, lacovelli R, Pantano F, Burattini L, Muzzonigro G, Berardi R, Santini D, Cascinu S. Risk of gastrointestinal events with sorafenib, sunitinib and pazopanib in patients with solid tumors: a systematic review and meta-analysis of clinical trials. Int J Cancer. 2014;135:763-73.

32. Brose MS, Frenette CT, Keefe SM, Stein SM. Management of sorafenib-related adverse events: a clinician's perspective. Semin Oncol. 2014:41(Suppl 2):S1-S16.

\section{Submit your next manuscript to BioMed Central and we will help you at every step:}

- We accept pre-submission inquiries

- Our selector tool helps you to find the most relevant journal

- We provide round the clock customer support

- Convenient online submission

- Thorough peer review

- Inclusion in PubMed and all major indexing services

- Maximum visibility for your research

Submit your manuscript at www.biomedcentral.com/submit
Biomed Central 